\title{
Towards a Forensic Psychological Evaluation of Juvenile Fire Setters: Parent Power
}

\author{
Ronn Johnson*, Andrea Fessler, Magdalene Wilhelm and Alejandra Stepensky
}

School, Family and Mental Health Professions, University of San Diego, USA

\begin{abstract}
Parents can provide information and insight critical to the understanding and treatment of clinical and forensic psychological issues presented by juvenile fire setters. Better inclusion of parents may improve treatment outcomes and thus promote public safety. Forensic assessments of juvenile fire setters (JFSB) often reveal emotional discord (e.g., anxiety, depression, social, trauma-related stress). The intentional features of a juvenile's case meet the $\mathrm{FBI}$ criteria for arson-related behaviors. Risk assessment by mental health professionals is imperative to try to anticipate the threat of repeat behavior. The ratings can then be used by a diverse group of professionals (e.g., psychosocial service providers, legal agencies, prosecutors, and probation officers). Parents are powerful (though sometimes reluctant) informants, who may require encouragement to assist mental health professionals craft better risk assessments. Information from parents can significantly influence the clinical and forensic service experiences of JFSs. This paper reviews the clinical and forensic matters relevant to understanding juvenile fire setters. The article examines risk assessment issues relative to using information provided by parents in the context of forensic work with juvenile fire setters. A section of the article is dedicated to the considering how the assessment of parents is used in the broader context of the forensic work with a juvenile. A recommended interview procedure for parents of juvenile fire setters is reviewed. Conclusions for forensic research and implications for forensic psychological practice are also highlighted.
\end{abstract}

Keywords: Alcoholism; Juvenile Fire Setters (JFS); Risk assessments

\section{Assessment and Intervention Issues for Fire Setters}

Internationally, Juvenile Fire Setters/Setting (JFS) constitutes an expensive, life-threatening problem for youth under the age of 19 [13]. Threats range from "playing" with matches, lighters, or portable torches to setting fires. All of the materials needed for setting fires are readily available to juveniles. For example, a case was referred for three juveniles that set fire to piles of paper products in a classroom, a hallway, and a boys' bathroom during school. The same boys were also involved in another fire that was set in a garage weeks earlier. These types of juvenile forensic psychology cases constitute a threat to public safety because the youth is usually involved in several fires before they are caught. The law enforcement response is costly in terms of resources and subsequently all interventions must be research based. For example, taking a juvenile fire setter for a ride on a fire truck is a documented approach even though it represents an inappropriate and even counterproductive response. The forensic likelihood of these youth reoffending increases because they frequently present with inadequate social skills, DSM5 Quad mental health-related problems (i.e., some combination of ADHD, PTSD, Conduct Disorder or ASD), family discord or parent psychopathology which can be summarized in an acronym (ISDQFP).

Legally, arson is defined as "any willful or malicious burning or attempt to burn, with or without intent to defraud, a dwelling, house, public building, motor vehicle or aircraft, or personal property of another" [4]. In the United States, twenty-two percent of school property fires were intentionally set and had estimated damages of over $\$ 100$ million [5,6]. Internationally, the costs are estimated to be in the billions. Arson is designated as a felony index crime. The term fire setting is often used to designate incidents with the absence of intent [7]. The juveniles referred by legal authorities to mental health service providers in these incidents can range in age from pre-school up to age $19[8,9]$.
Consistent with the DSM-5, the JFS risk point to mental health factors as a major contributing variable probably best assessed across several disorders (APA, 2013). Nevertheless, most youth in the correctional system for fire setting do not collectively meet criteria for one most commonly represented DSM-5 mental disorder [10]. Although, forensically the rates for JFS compared to non-JFS are disproportionately higher for antisocial personality disorder [11]. For example, mentally ill fire setters are more likely than non-mentally ill fire setters to have prior convictions for fire setting [12]. Alcoholism and substance abuse are also found in this JFS group [13-16].

A comprehensive approach (e.g., biopsychosocialcultural) is recommended for these juveniles because of the complexity of the inherent difficulties that complicate the risk assessment process for JFS. For example, psychohistorical information provided by parents must be weighed by mental health professionals to uncover a pattern of aggravating, motivational, and protective factors $[17,18]$. At the same time, ethnoracial factors assume a critical clinical forensic role in working with JFS cases and their parents. According to the DSM-5, a culturally-responsive explanatory models used must integrate concepts that provide greater clinical utility. Individual differences reflected in ethnicity and race can influence symptomatology which impacts the

*Corresponding author: Ronn Johnson, School, Family and Mental Health Professions, School of Education, University of San Diego, USA, Tel: (619) 2604702; E-mail: ronnjohncts@gmail.com

Received December 02, 2013; Accepted January 30, 2014; Published February 06, 2014

Citation: Johnson R, Fessler A, Wilhelm M, Stepensky A (2014) Towards a Forensic Psychological Evaluation of Juvenile Fire Setters: Parent Power. J Forensic Res 5 : 214. doi:10.4172/2157-7145.1000214

Copyright: (c) 2014 Johnson R, et al. This is an open-access article distributed under the terms of the Creative Commons Attribution License, which permits unrestricted use, distribution, and reproduction in any medium, provided the original author and source are credited. 
assessment methodology chosen for any forensic evaluation of parentchild activities [19].

\section{Evaluating Parental Perceptions of Dsyfunctional Child Actions}

Parents are the best knowledge keepers as well as instigators of the aggravating and resiliency biopsychosocialcultural factors for Juvenile Fire Setters (JFS). That is, there is evidence that reinforces the firm belief that parents function in a critical role in the developmental pathways (i.e., psychosocial and emotional) of their children [20]. For example, a JFS assessed with a developmental disorder is more likely than not to have the roots of these neurodevelopmental difficulties traced to any parent-related factors or other childhood experiences that provide unique clinical or forensic insights into functioning [21-24]. A logical question that arises relates to why is it desirable to collect this information from parents? One simple answer is because research indicates that the age that behavioral indicators occur could serve as a significant predictor of subsequent JSF-related behavior. In this case, the younger the onset of the JFS behavior the more likely is a neurodevelopmental factor operating that a parent would play a significant role during the appraisal process [19]. Put differently, the younger a child the more likely their JFS behavior can be assessed as influenced by any parent-related factors (e.g., inadequate supervision, parent psychopathology). Parents are important sources of information [25-27]. Nevertheless, many clinicians believe that parents are aware of and in many cases prone to downplay the extent of their child's JFSrelated behaviors. Therefore, parents should never be the sole source for JFS inputs in these types of forensic evaluations [28].

Two empirically-relevant questions have not been adequately addressed in the JFS research literature. First, which assessment approaches in the parental data gathering process can be used to identify markers for moderate to high-risk JFS cases? Second, in the aftermath of a JFS referral, to what extent can the absence of awareness, denied or minimized behavioral assessment data from parents be used to craft a composite risk assessment? For example, a forensic psychology case involving twin pre-school boys who were referred by the juvenile courts to the Burn Institute in San Diego (i.e., countywide forensic juvenile fire setter program) for a setting a neighbor's porch on fire. This referral is designated as the "SD Case" was made to the San Diego Burn Institutes Juvenile Fire Setter Research \& Intervention Center (JAERIC). The author supervised the risk assessment, psychological evaluation, and FATJAM Treatment (Forensic Assessment, Therapeutic Jurisprudence Assistance Model which is an evidenced-based CBT approach).

The SD Case is highlighted here because it illustrates the lower age range where JFS can occur as well as the problematic interactions between parental psychopathology and neurodevelopmental factors. A recap of the psychosocial history revealed that the twin's mother possessed neurodevelopmental problems and had received numerous child welfare service complaints for the poor supervision of her children. In the past the boys had burned themselves on the stove. During a clinical interview the mother reported that she frequently allowed her children to run naked in the front of their house, and lighters were left around the house-giving the boys easy access to them. She showered regularly with these boys. The forensic interview revealed several other fire setting incidents in the house since they were two-years-old. Parents are sometimes not aware of the role that they acquire in the course of JFS and the information that they provide, resist disclosing, or deny having clinical forensic significance of the risk assessment for determining future fire setting (e.g., low, medium or high risk).

Parenting issue markers are sometimes revealed during an initial forensic intake interview. For example, a forensic red flag is usually raised whenever the parents of an older JFS referral report that this was the first occurrence of fire setting for their child. This is quite often not the case. That is, some parents of older juveniles fail to accurately measure the frequency and onset of JFS incidents. For JFS cases, family affective factors often overlap with parenting supervision practices that can fuel the circumstances where fire setting or bomb making can occur. In the aforementioned JFS SD case, a clinical interview of the parent revealed several troublesome neurodevelopmental factors that the mother did not relate to her twins' negative behavioral or emotional functioning. A forensic mental health professional's assessment would pay particular attention to the parent's endorsements or lack thereofrelated to JFS behavior. For example, the mother of the twins failed to disclose relevant information about the children's JFS behavior orcriticaldetails about CPS actions taken against her. Parents have considerable amounts of information about their experiences and interactions during the course of time while raising their children [29]. The forensically-relevant information obtained from parents can be incomplete for an assortment of reasons. For instance, some parents internalize the circumstances where their child has been brought to the attention of the legal authorities by the fire setting or bomb making incident [30]. As a result, the parent may be more motivated to deny, distort, minimize, or simply be unaware of JSF issues related to their child. It is not unusual for another group of parents to externalize the JFS matter in order to shield authorities from knowing about their illadvised parenting practices [31]. These parents can be transparently defensive, openly hostile, and resentful of the organizational structure (e.g., not wanting to meet during school hours or condensing sessions, etc.) of the evidence-based forensic services they are being offered. For example, one parent with a history of juvenile hall contact, child protective services, and a bipolar mental disorder intentionally omitted responding to items on the MMPI2-RF she was administered. Gross inconsistencies were found between the information she provided when compared to her JFS referred child. A small group of parents may want to demand that the FATJAM treatment services (i.e., complete the entire program in a weekend or double up on the sessions) be reshaped to meet their immediate scheduling needs. The natural protective nature of some parents can result in them trying to shield their children from the legal consequences of their actions by withholding key information. To no surprise, the disposition of a parent can impact the course of treatment for any JFS. Unfortunately, the attitudes and behaviors of a parent can be unwittingly conveyed to the JFS in a way that compromises their ability to be motivated for the experience required for continuity of care that further enhances the benefits accrued from evidence-based treatments (FATJAM). Noncompliance with the treatment regimen can also result in the juvenile not being properly assessed for having a risk level of fire setting that is conducive to public safety [32]. It is important to show that there are less than a handful of evidence-based treatment programs across the country exclusively devote their clinical forensic services to JFScases. The vast majority of services for fire setters are limited educational in nature, non-mental health professionalsorthemental health providers delivering services are restricted to the occasional cases, they may be referred [32]. Despite the implied integrity caveats, a parent's endorsement represents a defining characteristic of their experience with the referred child that constitutes their subjective perceptions. The psychometric precision of the risk assessment rating for a JFS is assessed by some subjective 
perceptions. In the risk assessment process, ethically qualified mental health professionals rely upon the structural groundwork for the parent's endorsements.

\section{Conducting Risk Assessments Based on Parent's Information}

A risk assessment represents a critical public safety factor in any clinical forensic work with juvenile fire setters. That is, based on a number of forensically-relevant factors (e.g., history of previous fire setting incidents, parent psychopathology, gender, etc.) a juvenile can be evaluated as a low, medium or high risk for future fire setting. The rating is specifically relevant to parent psychopathology elements as well as the accuracy and detail of the information they furnish. Several parenting and family factors are linked to variables found useful in identifying data that may be important for the risk assessments performed for fire setting or bomb making [32-35]. The goal of risk assessment in current JFS practice is to identify juveniles who pose a high probability for reoffending or future fire setting. These juveniles are also likely to require individualized interventions [36,37]. For JFS, a risk assessment process means a examining the parent endorsements that function as strong predictors of their likelihood to reoffend. At the time of the referral, a parent or teacher are preferred sources for the historical experience with the JFS in question. From a forensic standpoint, the psycho-legal referral questions must also include other collaterals that offer relevant risk assessment information (i.e., regardless of concerns about the veracity of that information). All sources of information used in crafting the JFS clinical or forensic findings have limitations of applicability of risk assessment. The utility of the parent endorsements is maximized when used in a cumulative process that is part of a larger clinical or forensic intervention model [32].

A significant element of the risk assessment is dependent on the parent's information. Parents are usually in a essential position to evaluate the presence of any mental health disorders that they may have already been previously observed in their child. It is the responsibility of the forensic psychological examiner to appropriately utilize the information provided by parents in establishing a risk assessment rating. All JFS risk assessments focus on public safety or threat reduction efforts stemming from fire setting risks. The assessments conducted in these cases can also uncover additional clinical issues that, while important, they are not often the subject of treatment for JFS. Why, because treatment is focused on lowering the underlying public safety risk posed by the fire setting behavior as opposed to a clinical disorder. There are various assessment methods that can be used for securing information from parents. The parent assessment questions listed below were derived from the author's familiarity with the challenges posed by these types of cases over 20+ years of diverse forensic psychology practice and supervision experiences. The author also had an additional decade of peer-reviewed research and experience supervising and delivering services circumscribed to JFSs. The questions were formed through a review of a review of the juvenile fire setter research literature. The questions have been fieldtested by forensic mental health specialists at the Burn Institute of San Diego County. Additional field testing in other settings is strongly recommended as a future avenueof inquiry.

\section{JFS Parent Interview Questions}

* What changes have you noticed in your child's behavior? If so, when did you notice a differenceorchange?

* What specifically has changed about your child's behavior?

* How does your child spend time when not in school?

* How would you describe your child's behavior in school, at home, and in other settings?

* Please describe your child's interaction style with others (including family, friends/peers)?

* Have you or anyone else in your family noticed unusual burn marks or holes (to include: rugs/carpeting, clothing, furniture, etc.)?

* How much time does your child spend alone or unsupervised?

* Do you feel that your child receives a strange fascination with fire or chemicals? What leads you to say thatorto feel this way?

* Has your child ever been arrested or involved in the juvenile justice/court system? What were the circumstances?

* Is there a history of domestic violence, physical or sexual abuse, or substance use in your home?Please explain further (Figures 1 and 2).

The purpose of including the aforementioned table is to provide more of a structured information gathering method for JFS-related behavior. In summary, the aforementioned interview approach used for JFS indicates that parental endorsement is a relevant source of data for developing risk assessments. The clinical and forensic psychological results are directly impacted by parent's information because of their unique perspective on the juvenile's fire setting behaviors.

\section{Conclusions and Implications for Forensic Research and Practice}

The discussion here in this article has offered insights as to how the JFS evaluation approach with parents must be structured in a manner that facilitates a risk assessment that can be applied in a broader evidence-based treatment program. Parent factors function as a powerful source of influence in JFS cases. By default they must be included in the information gathering process for these types of forensic matters. For example, low parental warmth, parent-child conflict, poor supervision, ineffective or harsh discipline practices, and parents who themselves model antisocial behavior, can beleading risk factors for JFS related misconduct behaviors such as [38-42].

One of the goals of JFS research is to provide information that provides insights that extend the foundation of evidence-based practices (e.g., parent/youth observation techniques, prevention, resiliency factors, recognizing behavioral disorders). For example, there is a paucity of clinical or forensic information for small group fire setters as well as female fire setters. There is considerable ethnoracial diversity in the clinical and forensic psychological patterns of JFS that carries implications for assessment, diagnosis, and intervention. An expanded diagnostic framework of the DSM-5 (e.g., JFSB DSM5 Quad) in practice can function as a prism for clinical forensic case conceptualization and evidenced-based psychological interventions. Finally, the adoption of a generic culturally responsivesemi-structured parent interview approach would likely to fulfill the initial assessment screening needs for the juveniles encountered in these types of cases. 
Citation: Johnson R, Fessler A, Wilhelm M, Stepensky A (2014) Towards a Forensic Psychological Evaluation of Juvenile Fire Setters: Parent Power. J Forensic Res 5: 214. doi:10.4172/2157-7145.1000214

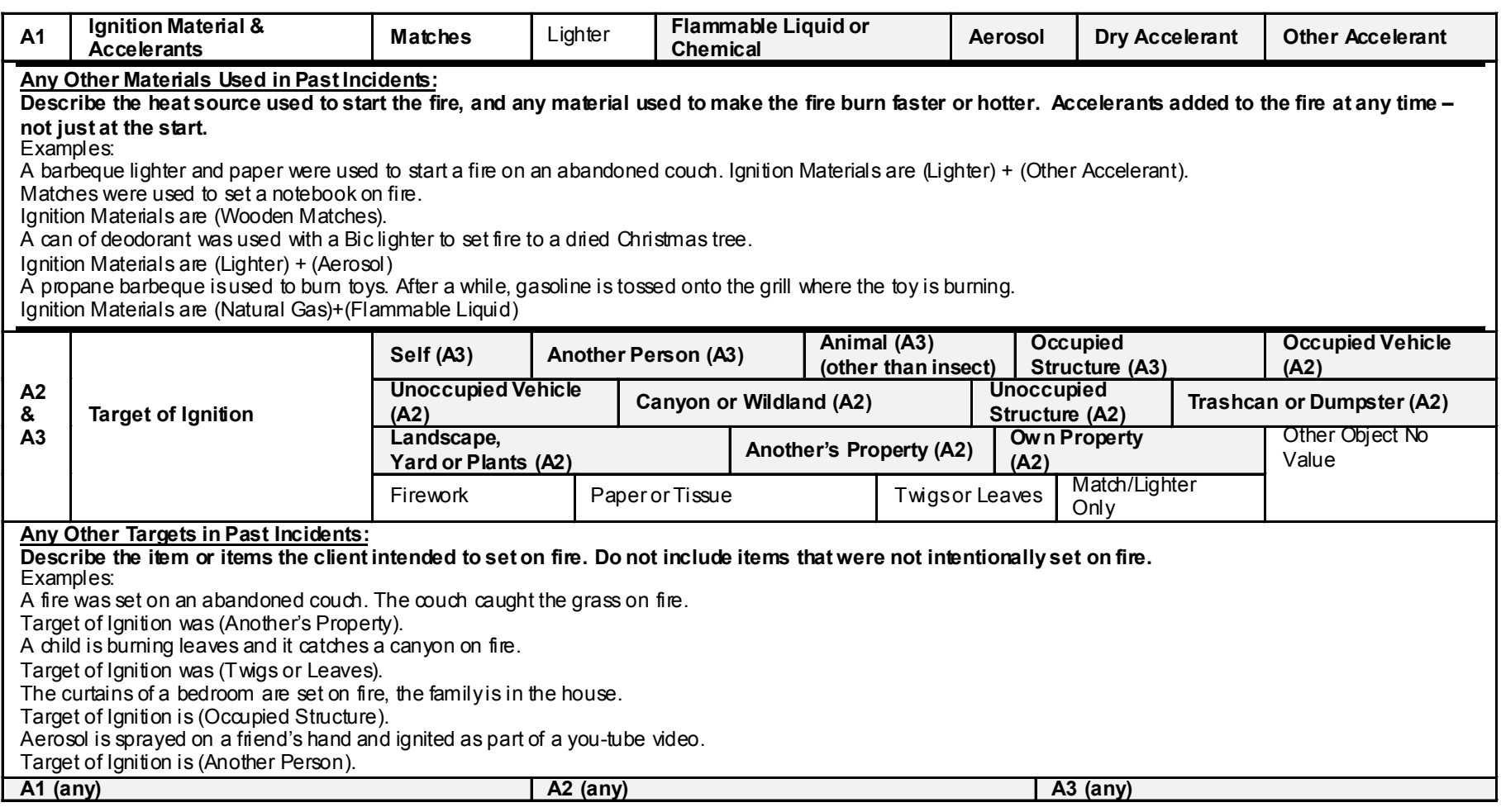

Figure 1: FAMJAM Parent Assessment Inventory (Damage Anticipation).

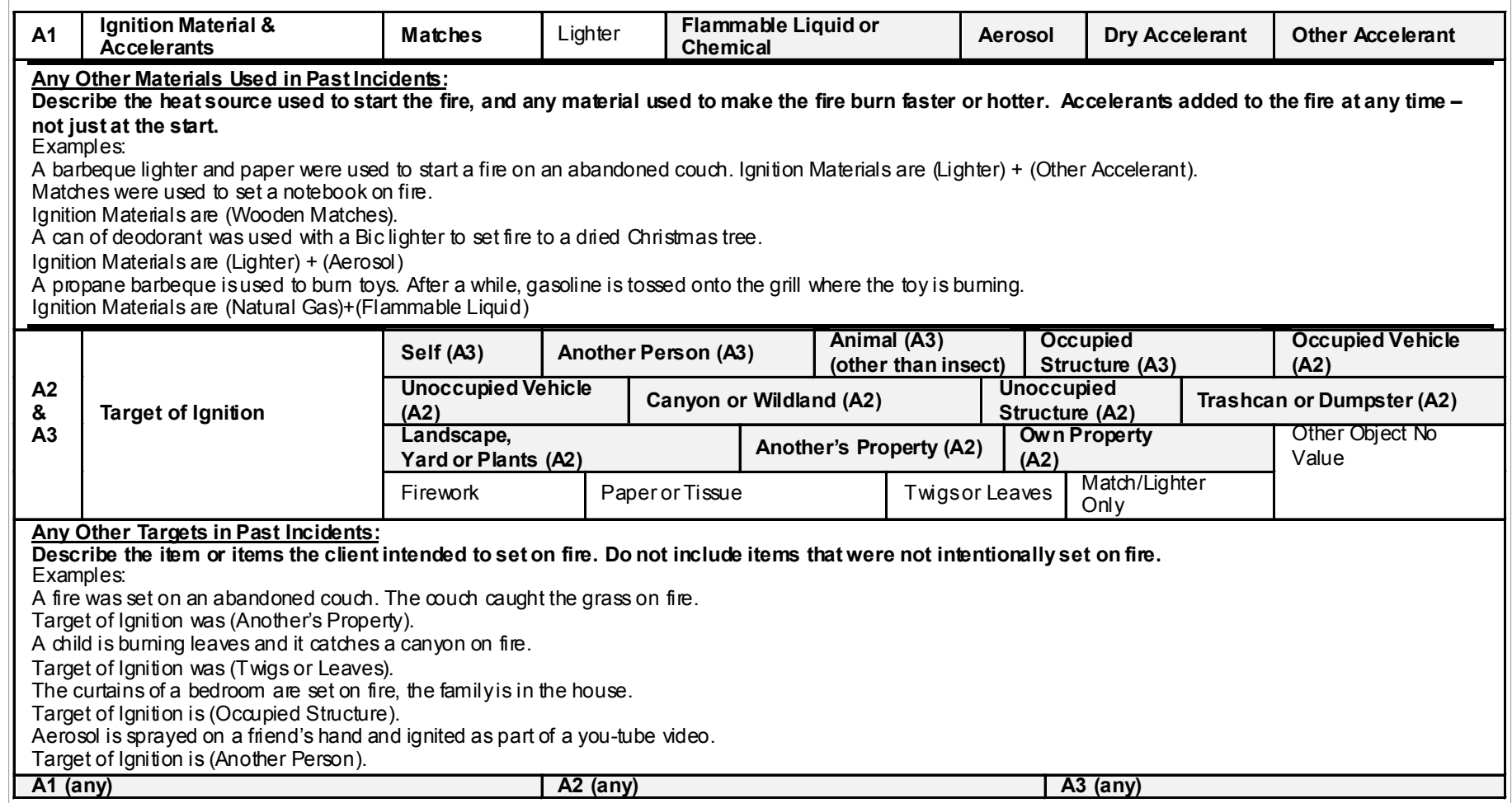

Figure 2: FATJAM Parent Assessment Inventory (Ignition Source). 
Citation: Johnson R, Fessler A, Wilhelm M, Stepensky A (2014) Towards a Forensic Psychological Evaluation of Juvenile Fire Setters: Parent Power. J Forensic Res 5: 214. doi:10.4172/2157-7145.1000214

Page 5 of 5

\section{References}

1. Brett $A$ (2004) 'Kindling theory' in arson: how dangerous are firesetters? Aust $N$ Z J Psychiatry 38: 419-425.

2. Doley R (2003) Pyromania: fact or fiction? British Journal of Criminology 43 : 797-807.

3. Epps K, Hollin CR (2000) Understanding and treating adolescent fire setters, In: Boswell G (ed.) Violent children and adolescents: asking the question why? London: Whurr: 36-55.

4. Federal Bureau of Investigation: Arson (2011) Crime in the United States.

5. Federal Emergency Management Agency (2004) The Fire Risk to Children: Topical Research Series. Washington DC: United States Fire Administration.

6. Flynn J (2007) US Structure Fires in Eating and Drinking Establishments. In Fire Analysis and Research Division. National Fire Protection Association: Quincy, MA.

7. Geller JL (1992) Pathological firesetting in adults. Int J Law Psychiatry 15: 283302

8. Federal Emergency Management Agency (2005) School Fires: Topical ResearchSeries. United States Fire Administration, Washington DC, USA.

9. Office of Juvenile Justice and Delinquency Prevention (OJJDP) November 1997. Reporting Crimes Against Juveniles. US Department of Justice, Washington DC, USA.

10. Johnson R, Beckenbach H, Kilbourne S (2013) Forensic psychological public safety risk assessment integrated with culturally responsive treatment for juvenile fire setters: DSM-5 implications. Journal of Criminal Psychology 3: 4964.

11. Vaughn MG, Fu Q, Delisi M, Wright JP, Beaver KM, et al. (2010) Prevalence and correlates of fire-setting in the United States: results from the National Epidemiological Survey on Alcohol and Related Conditions. Comp Psychiatry 51: 217-223

12. Jayaraman A, Frazer J (2006) Arson: a growing inferno. Med Sci Law 46: 295300.

13. Grant JE, Won Kim S (2007) Clinical characteristics and psychiatric comorbidity of pyromania. J Clin Psychiatry 68: 1717-1722.

14. Labree W, Nijman H, van Marle H, Rassin E (2010) Backgrounds and characteristics of arsonists. Int J Law Psychiatry 33: 149-153.

15. Ritchie EC, Huff TG (1999) Psychiatric aspects of arsonists. J Forensic Sci 44: 733-740.

16. Enayati J, Grann M, Lubbe S, Fazel S (2008) Psychiatric morbidity in arsonists referred for forensic psychiatric assessment in Sweden. Journal of Forensic Psychiatry \& Psychology 19: 139-147.

17. Hanson M, Mackay S, Atkinson L, Staley S (1995) Firesetting during the preschool period: assessment and intervention issues. CanJPsychiatry 40: 299-303.

18. Augimeri LK, Enebrink P, Walsh M, Jiang D (2000) Gender-specific childhood risk assessment tools: early assessment risk lists for boys (earl-20b) and girls (earl-21g) in Handbook of Violence Risk Assessment. Edited by Otto RK Douglas, KS. New York, Routledge-Taylor \& Francis Group.

19. American Psychiatric Association (2013) Diagnostic and Statistical Manual of Mental Disorders (DSM-5). Arlington, VA, USA

20. Hart CH, Newell LD, Olsen SF (2003) Parenting skills and social-communicative competence in childhood in Handbook of Communication and Social Interaction Skills. Edited by Greene, JO, Burleson BR, Lawrence Erlbaum Associates, Publishers, Mahwah, NJ, US.

21. Unnever JD, Cullen FT, Agnew R (2006) Why is "bad" parenting criminogenic? Implications rival theories. Youth Violence \& Juvenile Justice 4: 3-33.
22. Sullivan PM, Knutson JF (1998) The association between child maltreatment and disabilities in a hospital-based epidemiological study. Child Abuse \& Neglect 22: 271-288.

23. Reiter S, Bryen DN, Shachar I (2007) Adolescents with intellectual disabilities as victims of abuse. Journal of Intellectual Disabilities 11: 371-387.

24. Akbaş S, Ahmet T, Koray K, Ozan P, Tülay K, et al. (2013) Characteristics of Sexual Abuse in a Sample of Turkish Children With and Without Mental Retardation, Referred for Legal Appraisal of the Psychological Repercussions. Sexuality and Disability 27: 205-213.

25. Frick PJ, Viding E (2009) Antisocial behavior from a developmental psychopathology perspective. DevPsychopathol 21: 1111-1131.

26. Moffitt TE (1993) Adolescence-limited and life-course-persistent antisocial behavior: A developmental taxonomy. Psychol Rev 100: 674-701.

27. Odgers CL, Moffitt TE, Broadbent JM, Dickson N, Hancox RJ, et al. (2008) Female and male antisocial trajectories: From childhood origins to adult outcomes. DevPsychopathol 20: 673-716.

28. David CF, Kisner JA (2000) Do Positive Self-Perceptions Have a 'Dark Side'? Examination of the Link between Perceptual Bias. J Abnorm Child Psychol 28: 327-337.

29. Murphy SA, Chung IJ, Johnson LC (2009) Adolescent adjustment and patterns of parents' behaviors in early and middle adolescence. Res Nurs Health 19 530-555.

30. Slavkin ML (2000) What every nurse needs to know about ... juvenile firesetters A report of the Juvenile Firesetter Intervention Project. J PsychosocNursMent Health Serv 38: 6-17.

31. Kolko DJ, Kazdin AE (1992) The emergence and recurrence of child firesetting a one-year prospective study. J Abnorm Child Psychol 20: 17-37.

32. Dilorio C, Pluhar E, Belcher L (2003) Parent-child communication about sexuality: A review of the literature from 1980-2002. Journal of HIVIAIDS Prevention \& Education for Adolescents \& Children 5: 7-32.

33. Huebner AJ, Howell LW (2003) Examining the relationship between adolescent sexual risk-taking and perceptions of monitoring, communication, and parenting styles. Journal of Adolescent Health 33: 71-78.

34. Metzler CW, Noell J, Biglan A, Ary D, Smolkowski K (1994) The social context for risky sexual behavior among adolescents. J Behav Med 17: 419-438.

35. Perrino T, González-Soldevilla A, Pantin H, Szapocznik J (2000) The role of families in adolescent HIV prevention: a review. Clin Child FamPsychol Rev 3: 81-96.

36. Gottfredson SD, Moriarty LJ (2006) Statistical Risk Assessment: Old Problems and New Applications, Crime \& Delinquency 52: 178-200.

37. Kent R (2001) Data construction and data analysis for survey research Palgrave Publishers; New York, NY, USA.

38. DeMatteo DS, Marlowe DB, Festinger DS (2006) Secondary Prevention Services for Clients Who Are Low Risk in Drug Court: A Conceptual Model. Crime \& Delinquency 52: 114-134.

39. Dishion TJ, Patterson GR (1992) Age effects in parent training outcome. Behav Therapy 23: 719-729.

40. Farrington DP (1989) Early predictors of adolescent aggression and adult violence. Violence Vict 4: 79-100.

41. Hawkins DJ, Herrenkohl TI, Farrington DP, Brewer D, Catalano RF, et al. (2000) Predictors of youth violence. Juvenile Justice Bulletin (NCJ-179065) U.S. Dept. of Justice, Office of Juvenile Justice \& Delinquency Prevention, Washington, DC, USA

42. Henggeler SW (1989) Delinquency in adolescence. Newbury Park: Sage. 